\title{
Blocking methods to prevent non-specific adhesion of mesenchymal stem cells to titanium and evaluate the efficiency of surface functionalization: albumin vs poly(ethylene glycol) coating
}

Premio SIBB 2014

\author{
R. FRAIOLI ${ }^{1,2,3}$, J.M. MANERO ${ }^{1,2}$, J. GIL ${ }^{1,2}$, C. MAS-MORUNO ${ }^{1,2,3}$ \\ ${ }^{1}$ Biomaterials, Biomechanics and Tissue Engineering Group, Department of Materials Science \\ and Metallurgical Engineering, Technical University of Catalonia (UPC), Barcelona \\ ${ }^{2}$ Research Networking Centre in Bioengineering, Biomaterials and \\ Nanomedicine (CIBER-BBN), Campus Río Ebro, Edificio I+D, Zaragoza \\ ${ }^{3}$ Centre for Research in NanoEngineering (CRNE) - UPC, Barcelona
}

\begin{abstract}
Surface modification of biocompatible materials with biologically-active molecules is a well-known strategy to enhance the osteointegration of implantable devices. In order to evaluate the efficiency of these treatments, an in vitro study of cell behavior on the modified surface is usually carried out. A key point to evaluate the efficacy of this strategy is to avoid non specific protein adsorption by creating a non-fouling background. This blocking step ensures that the observed response of cells can be exclusively ascribed to the modification treatment applied. Several techniques are available to create this neutral background. Thus, the aim of this study is to compare two different blocking methods, namely adsorption of albumin from bovine serum (BSA) and grafting of small polyethylene glycol (PEG) chains to titanium, which is the gold standard metal for orthopedics and dentistry. To this end, titanium surfaces were coated with a recently synthesized cell adhesive peptide-based molecule and subjected to a blocking procedure with either BSA or PEG. Non-functionalized titanium samples were also blocked and used as controls. The biological response of human mesenchymal stem cells was evaluated by measuring the number of attached cells and studying the degree of cell spreading on the substrate. Both aspects of cell behavior are not affected significantly by the blocking method: cells adhere and spread significantly more on the functionalized samples, regardless of the blocking method used. This confirms that the surface feature that defines cell response is the presencelabsence of the biomolecule, and not the anti-fouling layer. These results, together with the reduction of variability of results observed in presence of a blocking layer, demonstrate the efficacy and necessity of blocking the surface. PEG grafting is demonstrated as effective as BSA coating in reducing nonspecific interactions and not hindering the effect of the biomolecule. However, taken into account the numerous advantages of a synthetic and customizable polymer chain over a complex natural protein, PEG blocking stands out as a very good alternative to albumin adsorption.
\end{abstract}

Keywords: BSA, PEG, surface functionalization, cell adhesion, anti-fouling.

Correspondencia:

Carlos Mas-Moruno

Email: carles.mas.moruno@upc.edu

Telephone: +34934010814

Fax: +34934016706 


\section{Introduction}

In the last two decades, many chemical and/ or physical modifications of implantable devices surfaces have been proposed as viable and straightforward strategies to stimulate the osteointegration of bioinert materials and, thus, increase the implant lifetime [1]. By creating a modified surface, capable of positively influencing the process of wound healing, the bulk properties of the material are not affected, while the response of the surrounding tissue to the implant is significantly enhanced, avoiding possible causes of failure, such as fibrous encapsulation and chronic inflammation. In the case of dental and permanent orthopedic implants, the aim of these modification treatments is to promote the osteointegration of the device, i.e. direct formation of new bone on the implant, giving structural and functional connection between the living tissue and the synthetic material, as defined originally by Brånemark in 1983 [2]. To this end, physical modifications of the surface, from random roughness [3] to highly controlled nanotopography modification [4], have been demonstrated to influence cell response and foster attachment and differentiation of bone-forming cells, namely osteoblasts, and other cell types $[5,6]$. A parallel field of studies has focused on chemical modification of the surface, whose aim is to anchor to the surface biologically-relevant molecules, such as proteins, recombinant protein fragments, or peptides [7]. Such molecules are able to interact with cells through numerous cell receptors and consequently guide cell fate. To test the efficacy of these chemical modifications, in vitro studies are generally carried out. Clearly, the fact that cell response will be solely dependent on the functionalization molecule is of paramount importance in these studies. However, cells may also interact in a non-specific manner with proteins from the culture medium adsorbed on the surface and with the surface itself. To avoid these sorts of unspecific interactions, an "inert" background is needed. Apart from preventing these interactions, the background has to be designed in such a way that the activity of biomolecule is affected as little as possible. Tra- ditionally, adsorption of albumin from bovine serum (BSA) has been used for this purpose, as BSA-coated surfaces have been demonstrated to have strong anti-fouling character [8]. More recently, polymer-based strategies, such as the use of polyethylene glycol (PEG), have been investigated as viable and more customizable alternatives to the use of proteins [9]. In this study, the BSA-blocking of Ti surfaces was compared to the grafting of PEG short chains as an alternative blocking method. Titanium was chosen as substrate since it is the most used material for orthopedic and dental applications, given its high biocompatibility and good mechanical properties [10]. The first is guaranteed by the stable oxide layer which spontaneously forms on the surface of the metal $\left(\mathrm{TiO}_{2} / \mathrm{TiO} / \mathrm{Ti}_{2} \mathrm{O}_{3}\right)$, and prevents corrosion and metallic ions diffusion into the tissues. As regards mechanical properties, Ti possesses lower elastic module than many other metallic materials, such as stainless steel or CoCr alloys, thus reducing stress shielding effects on surrounding bones [10]. Chemical functionalization of the metallic surface was chosen as a strategy to induce the growth of new bone at the implant/bone interface and thus improve the osteointegration of the synthetic device, as explained before. The treatment consisted of the anchoring of a recently synthesized biomolecule, which selectively binds to one of the integrin receptors of cells and thereby influences cell behavior, as recently demonstrated by us [11]. This system offers a proper substrate to study the efficacy of the functionalization strategy, and how the two blocking procedures affect the results of the in vitro study. Specifically, after characterizing the surface, adhesion of mesenchymal stem cells on the biomolecule-coated $\mathrm{Ti}$ was studied, in terms of the number and spreading of cells on the metallic surface. The surface was either non blocked or blocked with BSA or PEG coating. These three conditions were compared with three negative controls, consisting of uncoated $\mathrm{Ti}$, either blocked with one of the two methods or not. This experimental system allowed to simultaneously evaluate the effect of two variables: the presence of a bioactive molecule and of the anti-fouling layer. 


\section{Materials and methods}

\section{Preparation of titanium surfaces}

Cylindrical commercially pure (CP) Ti bars (diameter: $10 \mathrm{~mm}$ ) were provided by Technalloy S.A. (Sant Cugat del Vallès, Spain). Ti disks (thickness: $2 \mathrm{~mm}$ ) were prepared by turning, smoothed with silicon carbide grinding papers (Neuertek S.A., Eibar and Beortek S.A., AsuaErandio, Spain) and polished with suspension of alumina particles $(1 \mu \mathrm{m}$ and $0.05 \mu \mathrm{m}$ particle size) on cotton clothes. After obtaining smooth mirror-like surfaces, the disks were ultrasonically rinsed with cyclohexane, isopropanol, distilled water, ethanol and acetone and stored dried. The three bioinert surfaces (figure 2, upper line) were obtained by using either directly the polished Ti disks, coating with BSA, or grafting a short chain of PEG. The coating with BSA was done by soaking the disks in a $1 \%$ solution of BSA in phosphate buffered saline (PBS) at room temperature (RT) for $1 \mathrm{~h}$. A similar procedure was used to graft the PEG chains: Ti disks were soaked in a $500 \mu \mathrm{M}$ solution of $\mathrm{H}_{3} \mathrm{C}-(\mathrm{PEG})_{4}-\mathrm{SH}$ (MT-PEG, Thermo Scientific, Waltham, MA, USA ) (Figure 1) for $1 \mathrm{~h}$ at room temperature. The three bioactive surfaces (figure 2, lower line) were prepared by anchoring a previously synthesized [12] biomolecule via silanization of titanium. Prior to silanization, samples were passivated with $65 \%$ (v/v) $\mathrm{HNO}_{3}$ for $1 \mathrm{~h}$ and then ultrasonically cleaned with MilliQ water, ethanol and acetone. Straight after the oxidizing treatment, the surfaces were silanized by immersing samples in 2\% (v/v) APTES (Sigma-Aldrich, St. Louis, MO, USA) in anhydrous toluene (Sigma-Aldrich) at $70{ }^{\circ} \mathrm{C}$ for $1 \mathrm{~h}$ under nitrogen atmosphere, and then ultrasonically rinsed with toluene for $5 \mathrm{~min}$. Next, samples were rinsed with MilliQ water, ethanol and acetone. The obtained surface was finally cured at $120{ }^{\circ} \mathrm{C}$ for 5 min. In order to couple the crosslinking agent $\mathrm{N}$-succinimidyl-3-maleimidopropionate (SMP) (Alfa Aesar, Karlsruhe, Germany), disks were soaked in 7.5 M solution in N,N-dimethylformamide (DMF) for $1 \mathrm{~h}$ at RT and rinsed with DMF, distilled water, ethanol and acetone afterwards. Both silanization and SMP coupling were performed under agitation and were followed by rinsing with MilliQ water, ethanol and acetone. The immobilization of the biomolecule was performed by first dissolving it in PBS at $100 \mu \mathrm{M}$ and $\mathrm{pH}$ 6.5, and then depositing 100 $\mu \mathrm{L}$ of these solutions overnight on samples at RT. Two of the three bioactive conditions were subsequently blocked with BSA or MT-PEG, as explained before. Surfaces were named as represented in Figure 2.

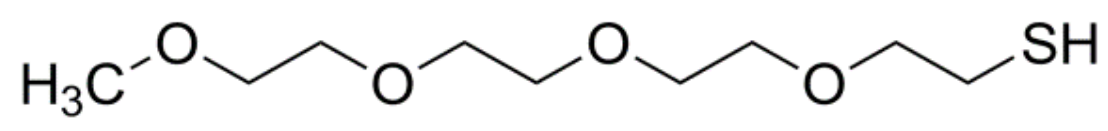

Figure 1. Chemical structure of the blocking molecule of PEG, which has a thiol group on one end, serving as anchor to the surface, and a methyl group on the other end, which has no specific biological effect.

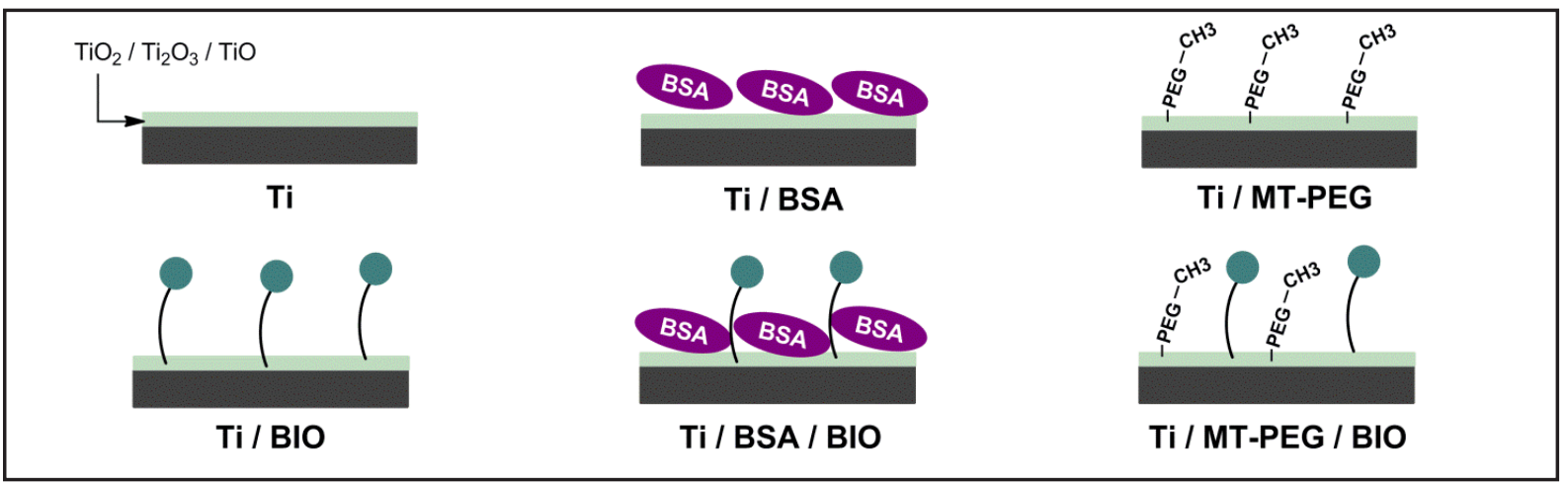

Figure 2. Schematic representation of the surfaces: three bioinert Ti surfaces (upper line) are compared with three functionalized surfaces, where a bioactive molecule (green circle) has been grafted. 
Characterization of surface physicochemical properties

The polishing treatment of Ti disks is intended to reduce surface roughness and generate a smooth surface, serving as a substrate for all conditions. To measure this reduction white light interferometry in vertical scanning interferometry mode (Wyko NT9300 Optical Profiler, Veeco Instruments, New York, NY, USA) was used before and after the polishing procedure. The average roughness $(\mathrm{Ra})$ of each sample was measured in three randomly chosen points of the disk. Data were analyzed with Wyko Vision 4.10 software (Veeco Instruments). Moreover, the sessile drop method was used to measure static contact angle of ultrapure Milli-Q water and diiodomethane (volume of wetting liquids: $1 \mu \mathrm{L})$ (Contact Angle System OCA15 plus, Dataphysics, Filderstadt, Germany), allowing the calculation of surface energy with Young-Laplace and Owen-Wendt equations.[13] Contact angle values of 3 drops per sample were obtained using Laplace-Young fitting with SCA 20 software (Dataphysics).

\section{Cell culture}

Human mesenchymal stem cells (hMSCs) were cultured in Advanced Dulbecco Modified Eagle Medium (DMEM) supplemented with $10 \%(\mathrm{v} / \mathrm{v})$ fetal bovine serum (FBS), $50 \mathrm{U} / \mathrm{mL}$ penicillin, $50 \mu \mathrm{g} / \mathrm{mL}$ streptomycin and $1 \%(\mathrm{w} / \mathrm{v}$ ) L-glutamine. Cells were kept at $37^{\circ} \mathrm{C}$, in a humidified atmosphere with $5 \%(\mathrm{v} / \mathrm{v}) \mathrm{CO}_{2}$ and changing culture medium twice a week. Upon reaching $70 \%$ confluence, cells were detached by trypsin-EDTA and subcultured into a new flask. Cells at passages between 2 and 5 were used to carry out all the experiments. All reagents were purchased from Sigma-Aldrich, unless otherwise stated.

\section{Cell adhesion}

Cell attachment on Ti surfaces was evaluated after $6 \mathrm{~h}$ of incubation in serum-free medium by quantification of cell number via enzymatic assay. Prior to seeding, samples were rinsed three times with PBS and either left untreated, blocked in $1 \%(\mathrm{w} / \mathrm{v})$ BSA or coated with MT-PEG as explained above. Ti disks were moved to 48well plates, cells were seeded at $10 \times 10^{3}$ cells/
$\mathrm{mL}$ and incubated at $37{ }^{\circ} \mathrm{C}$ and $5 \%(\mathrm{v} / \mathrm{v}) \mathrm{CO}_{2}$ containing atmosphere. $6 \mathrm{~h}$ post-seeding nonadherent cells were washed off by gently rinsing with PBS, and attached cells were lysed with 300 $\mu \mathrm{L} /$ disk mammalian protein extraction reagent (M-PER). Enzymatic activity of lactate dehydrogenase (LDH) was quantified by colorimetric assay (Cytotoxicity Detection Kit (LDH), Roche Diagnostics, Mannheim, Germany), using a multimode microplate reader (Infinite M200 PRO, Tecan Group Ltd., Männedorf, Switzerland).

Immunofluorescence analysis of cell morphology

hMSCs cells were plated on samples as previously explained. Cells were allowed to attach for $6 \mathrm{~h}$ in serum-free medium, and subsequently fixed with paraformaldehyde (PFA, 4\% w/v in PBS) for $20 \mathrm{~min}$, permeabilized with $500 \mu \mathrm{L} /$ disk of $0.05 \%(w / v)$ Triton X-100 in PBS for 20 min and blocked with $1 \%$ BSA (w/v) in PBS for $30 \mathrm{~min}$. Actin fibers and nuclei were stained by incubating with rhodamine-conjugated phalloidin (1:300, in Triton $0.05 \%$ (w/v) in PBS) for $1 \mathrm{~h}$ and with 4,6-diamidino-2-phenylindole (DAPI) $(1: 1000$, in PBS-Glycine $20 \mathrm{mM})$ for $2 \mathrm{~min}$ at RT in the dark, respectively. Ti disks were examined under a fluorescence inverted microscope (AF7000, Leica, Germany) and images processed using Fiji/Image-J package.

\section{Results and discussion}

\section{Surface characterization}

Cell behavior is deeply influenced by the microenvironment. Specifically, anchorage dependent cells, like osteoblasts and MSCs, respond to the set of chemical, topographical, mechanical, and biological features of their substrate $[3,14-$ 16]. In this study, we focused on the chemical composition of the surface, i.e. on the effect of the biomolecules anchored to the surface. To analyze the effect of this specific feature of the surface, and exclude the effect of other parameters, it is important to provide that all surfaces compared have the highest homogeneity as possible, in terms of topography, wettability and stiffness, in this case. Stiffness of all samples will be clearly the same, as cells sense the hard metallic surface in all conditions. As regards topogra- 
phy, smooth mirror-like Ti surfaces have been chosen as surface of reference, as the biological effect of the surface-bound biomolecules has been proved to be more evident on smoother substrates than on rougher ones [17]. White light interferometry was used to monitor the reduction of roughness after the polishing process. Passing from the turned surface to the polished one, arithmetic roughness is highly reduced, and roughness is not modified by the passivation protocol (Table 1), nor by any subsequent step of the functionalization protocol.

Hydrophilicity of the surface is slightly increased throughout the functionalization process (Figure 3). Contact angle of diiodomethane remains almost constant throughout the steps of the coating (not shown). Overall, surface energy spans a small range of values, which unlikely influences short-time cell response. On this regard, Kennedy et al. demonstrated that cell adhesion after $8 \mathrm{~h}$ of incubation on substrate with very different superficial energy was not affected [14].

\section{Effect of the blocking protocol on cell attachment} and spreading

In order to study the effect of the blocking method on the inert and the bioactive surfaces (Fig. 1), the number of attached cells and the projected cell area after short incubation time have been measured. After preparing surfaces as described above, hMSCs were plated and incubated on Ti for 6 hours. All three inert Ti surfaces support poor cell adhesion. Nevertheless, blocked conditions (Ti/BSA and Ti/MTPEG) display a tendency toward lower adhesion efficiency, compared to the inert untreated $\mathrm{Ti}$. This effect is probably due to the presence of the anti-fouling layer of BSA or MT-PEG. As a matter of fact, the small polymer chain has a methyl group at the end of the chain, which is known to have no specific biological effect on cells. Apart from that, PEG polymers are known to exert an anti-fouling effect mainly through two mechanisms: steric repulsion and/or hydratation/water structuring [18]. The origin of the first one is related to the resistance of polymer chains to compression: any protein approaching the PEG-coated surface will cause an increase in the local concentration of polymer chains, which corresponds to a reduction of the entropy of the system and, consequently, yields to an increase in free energy $(\Delta G)$. Hence, a repulsive energy of interaction is generated. Moreover, compression of the chains also generates an elastic term, which also contributes to the repulsive energy. The sum of these terms has to be greater in magnitude than the attractive electrostatic or van der Waals interaction. In the case of polymers, repulsive energy is higher than these attractive components, thus justifying their use as anti-fouling coatings. However, it can be noted that both repulsive components depend on the molecular weight (MW) of the polymer and on the grafting density achieved, with higher concentration and higher MW usually corresponding to higher repulsion. In this case these two variables were not taken into account, since only one type of PEG was used. The BSA coating is also known to serve as an effective blocking treatment, since it has been demonstrated that protein absorption is highly reduced on albumin [8], making it the routine blocking treatment in numerous studies $[11,19,20]$. Differently from blocked surfaces, the untreated inert Ti surface presents very high variability: as no blocking of the surface is done, cells adhere non-specifically to the metallic material, giving a response that is scarcely predictable.

Anchoring the biomolecule gives the surface much better adhesive properties, since all

\begin{tabular}{|c|c|}
\hline Sample & $\mathrm{R}_{\mathrm{a}}(\mathrm{nm})$ \\
\hline Not polished $\mathrm{Ti}$ & $493.7 \pm 72.1$ \\
\hline Polished $\mathrm{Ti}$ & $14.6 \pm 1.5$ \\
\hline $\mathrm{HNO}_{3}$ passivated $\mathrm{Ti}$ & $14.3 \pm 2.1$ \\
\hline
\end{tabular}

Table 1. Arithmetic roughness of the Ti surface before and after the polishing treatment and after the passivation procedure. Values are presented as mean \pm standard deviation. 
three bioactive surfaces ( $\mathrm{Ti} / \mathrm{BIO}, \mathrm{Ti} / \mathrm{BSA} / \mathrm{BIO}$ and Ti/MT-PEG/BIO) support a statistically higher $(\mathrm{p}<0.05)$ adhesion, compared to all inert conditions, as expected [11]. However, the blocking treatments do have a small effect on adhesion efficiency, lowering it, even though the decrease is not statistically significant. This is of paramount importance in the study of a novel functionalization molecule: in order to evaluate the efficacy of the biomolecule, no significant reduction in activity has to be caused by the blocking treatment, as this would mislead in drawing conclusions.

Furthermore, an immunofluorescence analysis was carried out to study the quality of adhesion. To this end, actin cytoskeleton was stained with

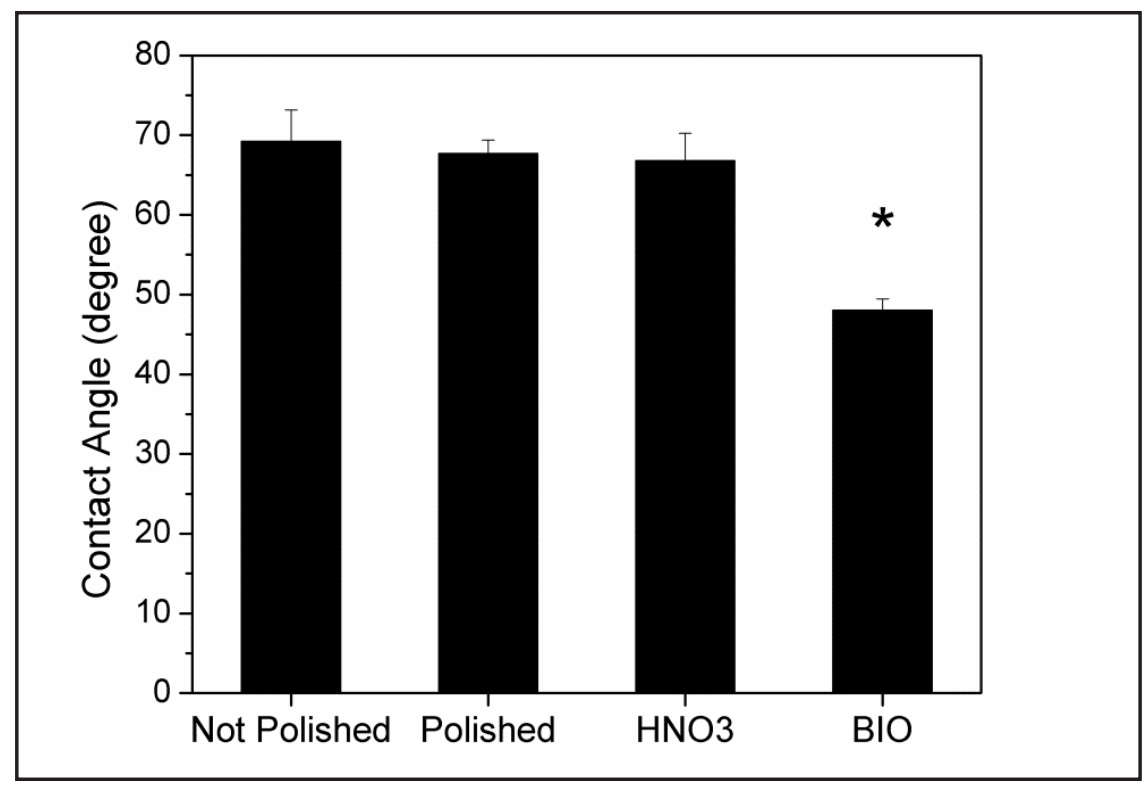

Figure 3. Water contact angle on Ti surface throughout the functionalization process. ${ }^{*} \mathrm{p}<0.05$ compared to the unlabeled columns by ANOVA.

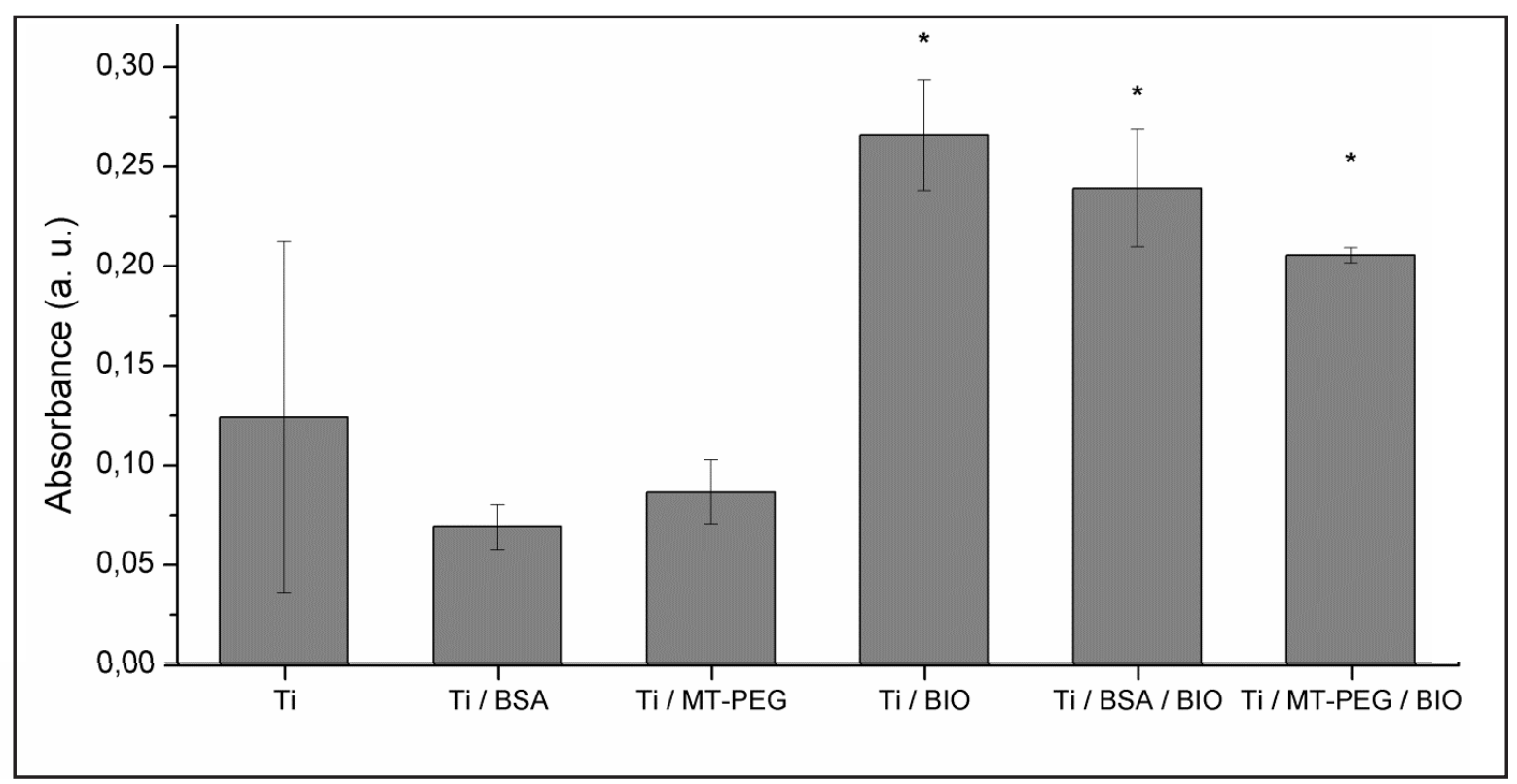

Figure 4. Absorbance of lactate dehydrogenase (LDH), which is directly proportional to the number of cells attached to the Ti surface. Results are mean values and bars represent standard deviation. * $\mathrm{p}<0.05$ compared to unlabeled columns by ANOVA. 
rhodamine-conjugated phalloidin after 6 hours of incubation on the metallic surfaces. The spreading of hMSCs on the metallic surfaces is either low, on uncoated $\mathrm{Ti}$, or high, on functionalized surfaces, as observed for cell attachment. Thus, cells do not spread on inert Ti conditions, both unblocked and BSA or PEG blocked samples, while they display a high projected area on all bioactive conditions. These results confirm that the blocking procedure is not modifying significantly the surface features, which are still governed by the absence/presence of a bioactive molecule.

Taken together, results demonstrate the validity of both blocking methods. However, several intrinsic features of the blocking molecules have to be taken into account. Being a protein, BSA is much more sensitive to temperature and $\mathrm{pH}$ variations, which may lead to the denaturation of the protein, while a polymer as PEG is not affec- ted in the range of temperature and $\mathrm{pH}$ typically encountered. Furthermore, a synthetic polymer chain is completely customizable: both the anchor and the terminal group of the chain can be substituted to suit the specific system studied. For instance, even bioactivity can be attained by simply grafting an active receptor-binding molecule to the end of the PEG chain, obtaining anti-fouling and cell adhesive properties at the same time [7]. Also the combination of several PEG chains to form the so-called polymer brushes is an alternative to increase the local density of chains and, thereby, the anti-fouling character of the coating [21]. Everything considered, polymer blocking methods offer a much more controlled and adjustable system, compared to complex and scarcely controlled natural macromolecules, and thus constitute a better alternative to the traditional albumin blocking methods.

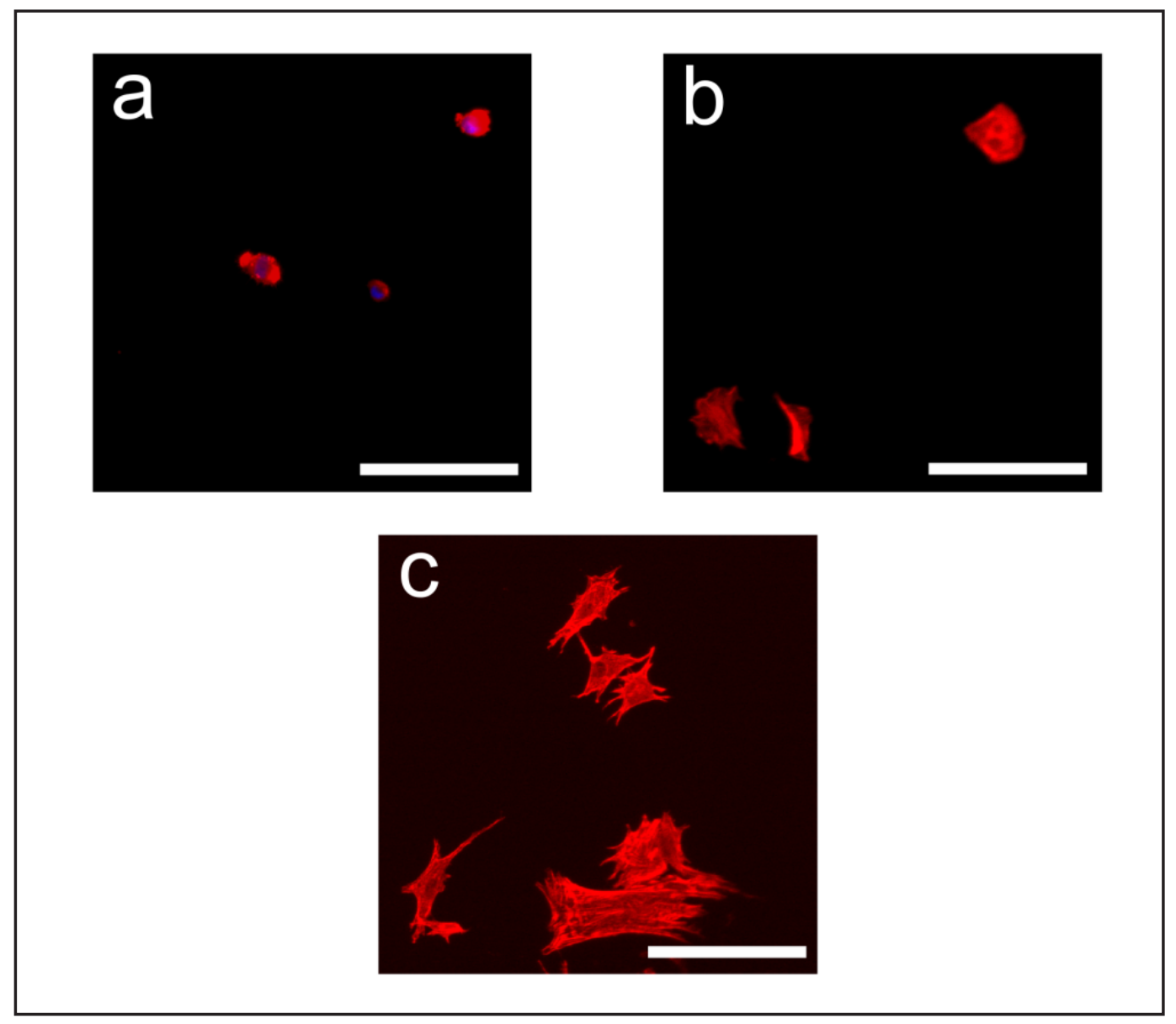

Figure 5. Spreading of hMSCs cell on the BSA-blocked Ti (a), MT-PEG-blocked Ti (b), and BSAblocked Ti coated with the biomolecule (c). Cells do not spread on both blocked surfaces, regardless of the blocking method, nor on the inert Ti (not shown). However, when the biomolecule is anchored to the surface, cells are able to spread completely either without blocking (not shown), in presence of BSA (c), or MT-PEG (not shown). Bar: $50 \mu \mathrm{m}$. 


\section{Conclusions}

A comparison between two different blocking methods, a more traditional one with albumin, and a polymer-based one, has been presented in this study. The study has focused on two aspects, namely the efficiency in having more controlled response on inert surfaces, and the ability of the anti-fouling layer not to hinder the effect of an active biomolecule anchored on the material. Both blocking methods have been proved viable to study the efficacy of chemical functionalization strategies on metal, providing a more controlled response and not interfering with the effect of the biomolecule. The study only focused on two standard biological assays, i.e. attachment and evaluation of cell projected area, since these are the most common in vitro studies, especially in preliminary studies evaluating the viability of a novel functionalization approach. This kind of studies aims at obtaining a clear and unequivocal biological result, hence, it is crucial that nothing interferes with the experimental outcome, dampening the effect of the studied molecule. Generally, our data definitely support the use of a blocking procedure, either with albumin coating, or with the grafting of small PEG chain, since:

- Lower variability and, thus, higher control over adhesive processes is obtained, as evident from the reduction of standard deviation of both blocked inert Ti conditions, compared to the unblocked inert $\mathrm{Ti}$;

- The positive effect on attachment resulting from the anchoring of the biomolecule is not affected significantly by the blocking step, meaning that the functionalization treatment is not invalidated by the presence of the anti-fouling layer;

- Cells are not able to spread on none of the inert surfaces, while they spread on all bioactive surfaces, again demonstrating the non-invasiveness of the blocking step over this other aspect of cell response;

- Although not demonstrated in this study, it is known that the polymer molecules do not degrade in the physiological environment, while proteins, such as BSA, are prone to denaturation due to enzymes, sharp changes in temperature or $\mathrm{pH}$. Moreover, $\mathrm{PEG}$ is known to have anti- bacterial effect [22], which would add another advantage to this type of blocking procedure;

On the whole, results confirm the viability of both techniques to block non-specific interaction with the substrate. Nonetheless, considering the non degradability, antibacterial properties, and the spectrum of chemical modification that can be easily applied to synthetic polymers, PEG-blocking seems a better blocking strategy for surface functionalization studies.

\section{References}

1. Tejero R, Anitua E, Orive G. Toward the biomimetic implant surface: Biopolymers on titaniumbased implants for bone regeneration. Prog Polym Sci 2014;39:1406-47. doi:10.1016/j.progpolymsci.2014.01.001.

2. Brånemark R, Brånemark $\mathrm{PI}$, Rydevik $\mathrm{B}$, Myers RR. Osseointegration in skeletal reconstruction and rehabilitation: a review. J Rehabil Res Dev 2001;38:175-81.

3. Anselme K, Bigerelle M. Topography effects of pure titanium substrates on human osteoblast long-term adhesion. Acta Biomater 2005;1:211-22. doi:10.1016/j.actbio.2004.11.009.

4. Sjöström T, McNamara LE, Meek RMD, Dalby MJ, Su B. 2D and 3D nanopatterning of titanium for enhancing osteoinduction of stem cells at implant surfaces. Adv Healthc Mater 2013;2:1285-93. doi:10.1002/adhm.201200353.

5. Lincks J, Boyan BD, Blanchard CR, Lohmann CH, Liu Y, Cochran DL, et al. Response of MG63 osteoblast-like cells to titanium and titanium alloy is dependent on surface roughness and composition. Biomaterials 1998;19:2219-32.

6. Nikkhah M, Edalat F, Manoucheri S, Khademhosseini A. Engineering microscale topographies to control the cell-substrate interface. Biomaterials 2012;33:5230-46. doi:10.1016/j.biomaterials.2012.03.079.

7. Rodda AE, Meagher L, Nisbet DR, Forsythe JS. Specific control of cell-material interactions: Targeting cell receptors using ligand-functionalized polymer substrates. Prog Polym Sci 2013. doi:10.1016/j. progpolymsci.2013.11.006.

8. Barisci J, Hughes D, Minett a, Wallace G. Characterisation and analytical use of a polypyrrole electrode containing anti-human serum albumin. Anal Chim Acta 1998;371:39-48. doi:10.1016/S00032670(98)00277-3.

9. Dalsin J, Messersmith P. Bioinspired antifouling polymers. Mater Today 2005:38-46.

10. Geetha M, Singh AK, Asokamani R, Gogia AK. Ti based biomaterials, the ultimate choice for orthopaedic implants - A review. Prog Mater Sci 2009;54:397425. doi:10.1016/j.pmatsci.2008.06.004. 
11. Fraioli $R$, Rechenmacher F, Neubauer S, Manero JM, Gil J, Kessler H, et al. Mimicking Bone Extracellular Matrix: Integrin-Binding Peptidomimetics Enhance Osteoblast-like Cells Adhesion, Proliferation, and Differentiation on Titanium. Colloids Surfaces B Biointerfaces 2015. doi:10.1016/j.colsurfb.2014.12.057.

12. Rechenmacher F, Neubauer S, Polleux J, MasMoruno C, De Simone M, Cavalcanti-Adam EA, et al. Functionalizing $\alpha v \beta 3$ - or $\alpha 5 \beta 1$-selective integrin antagonists for surface coating: a method to discriminate integrin subtypes in vitro. Angew Chem Int Ed Engl 2013;52:1572-5. doi:10.1002/ anie.201206370.

13. Owens DK, Wendt RC. Estimation of the surface free energy of polymers. J Appl Polym Sci 1969;13:1741-7.

14. Kennedy SB, Washburn NR, Simon CG, Amis EJ. Combinatorial screen of the effect of surface energy on fibronectin-mediated osteoblast adhesion, spreading and proliferation. Biomaterials 2006;27:381724. doi:10.1016/j.biomaterials.2006.02.044.

15. Schwab EH, Halbig M, Glenske $K$, Wagner A-S, Wenisch S, Cavalcanti-Adam E a. Distinct effects of RGD-glycoproteins on Integrin-mediated adhesion and osteogenic differentiation of human mesenchymal stem cells. Int J Med Sci 2013;10:1846-59. doi:10.7150/ijms.6908.

16. Rowlands AS, George $P$ a, Cooper-White JJ. Directing osteogenic and myogenic differentiation of MSCs: interplay of stiffness and adhesi- ve ligand presentation. Am J Physiol Cell Physiol 2008;295:C1037-44. doi:10.1152/ajpcell.67.2008.

17. Mas-Moruno C, Dorfner PM, Manzenrieder F, Neubauer S, Reuning U, Burgkart R, et al. Behavior of primary human osteoblasts on trimmed and sandblasted Ti6Al4V surfaces functionalized with integrin $\alpha \mathrm{v} \beta 3$-selective cyclic RGD peptides. J Biomed Mater Res A 2013;101:87-97. doi:10.1002/ jbm.a.34303.

18. Vermette $\mathbf{P}$, Meagher L. Interactions of phospholipid-and poly (ethylene glycol)-modified surfaces with biological systems: relation to physico-chemical properties and mechanisms. Colloids Surfaces B Biointerfaces 2003;28.

19. Mas-Moruno C, Fraioli R, Albericio F, Manero JM, Gil FJ. Novel peptide-based platform for the dual presentation of biologically active peptide motifs on biomaterials. ACS Appl Mater Interfaces 2014;6:6525-36. doi:10.1021/am5001213.

20. Frith JE, Mills RJ, Cooper-White JJ. Lateral spacing of adhesion peptides influences human mesenchymal stem cell behaviour. J Cell Sci 2012;125:31727. doi: $10.1242 /$ jcs. 087916.

21. Moroni L, Gunnewiek MK, Benetti EM. Polymer Brush Coatings Regulating Cell Behavior: Passive Interfaces Turn Into Active. Acta Biomater 2014:112. doi:10.1016/j.actbio.2014.02.048.

22. Ding X, Yang C, Lim T, Hsu L, Engler A. Antibacterial and antifouling catheter coatings using surface grafted PEG- b-cationic polycarbonate diblock copolymers. Biomaterials 2012;33. 